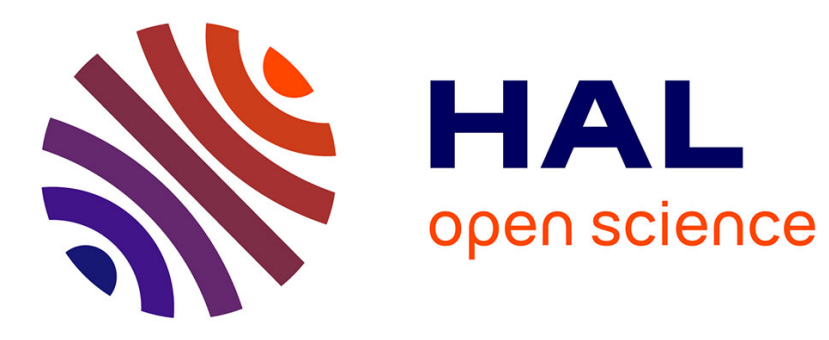

\title{
Hyperquaternions: An Efficient Mathematical Formalism for Geometry
}

Patrick Girard, Patrick Clarysse, Romaric Pujol, Robert Goutte, Philippe Delachartre

\section{- To cite this version:}

Patrick Girard, Patrick Clarysse, Romaric Pujol, Robert Goutte, Philippe Delachartre. Hyperquaternions: An Efficient Mathematical Formalism for Geometry. Geometric Science of Information. 4th International Conference (GSI 2019), Frank Nielsen; Frédéric Barbaresco, Aug 2019, Toulouse, France. pp.116-125, 10.1007/978-3-030-26980-7_13 . hal-02276429

\section{HAL Id: hal-02276429 \\ https://hal.science/hal-02276429}

Submitted on 4 Jan 2021

HAL is a multi-disciplinary open access archive for the deposit and dissemination of scientific research documents, whether they are published or not. The documents may come from teaching and research institutions in France or abroad, or from public or private research centers.
L'archive ouverte pluridisciplinaire HAL, est destinée au dépôt et à la diffusion de documents scientifiques de niveau recherche, publiés ou non, émanant des établissements d'enseignement et de recherche français ou étrangers, des laboratoires publics ou privés. 


\title{
Hyperquaternions: An Efficient Mathematical Formalism for Geometry
}

\author{
Patrick R. Girard ${ }^{1[0000-0001-8556-1816]}$, Patrick Clarysse ${ }^{1[0000-0002-5495-7655]}$, \\ Romaric Pujol ${ }^{2}$, Robert Goutte ${ }^{1}$, and Philippe \\ Delachartre $^{1[0000-0003-0492-1846]}$ \\ 1 Univ Lyon, INSA-LYON, Université Claude Bernard Lyon 1, UJM-Saint Etienne, \\ CNRS, Inserm, CREATIS UMR 5220, U1206, F-69621 LYON, France \\ patrick.girard@creatis.insa-lyon.fr \\ 2 Pôle de Mathématiques, INSA-Lyon \\ Bât. Léonard de Vinci, 21 avenue Jean Capelle \\ F-69621 Villeurbanne, France
}

\begin{abstract}
Hyperquaternions being defined as a tensor product of quaternion algebras (or a subalgebra thereof), they constitute Clifford algebras endowed with an associative exterior product providing an efficient mathematical formalism for differential geometry. The paper presents a hyperquaternion formulation of pseudo-euclidean rotations and the Poincaré groups in $n$ dimensions (via dual hyperquaternions). A canonical decomposition of these groups is developed as an extension of an euclidean formalism and illustrated by a $5 D$ example. Potential applications include in particular, moving reference frames and machine learning.
\end{abstract}

Keywords: Quaternions · Hyperquaternions · Pseudo-euclidean rotations · Poincaré groups · Canonical decomposition.

\section{Introduction}

Clifford algebras allow an excellent representation of pseudo-euclidean rotations which are important symmetry groups of physics [1-4]. A decomposition of these groups into orthogonal, commuting planar rotations is called a canonical decomposition. Various canonical decompositions have been developed which deal with either specific rotations or dimensions and are often expressed in terms of matrices $[5,6]$. In a recent paper, we have introduced a hyperquaternion formulation of Clifford algebras and applied them to the unitary and unitary symplectic groups [7]. Here, we consider pseudo-euclidean rotations and the Poincaré groups in $n$ dimensions (via dual hyperquaternions). A canonical decomposition of these groups is developed within that framework as an extension of an euclidean formalism introduced by Moore $[8,9]$. After a short presentation of hyperquaternions and multivectors, we derive the pseudo-euclidean rotations and the canonical decomposition. Then we go on to the Poincaré groups and a $5 D$ example. Potential applications are moving reference frames and machine learning [10] 
Table 1. Biquaternion Multivector Structure

$$
\begin{array}{|l|l|l|l|}
\hline 1 & i=e_{3} e_{2} & j=e_{1} e_{3} & k=e_{2} e_{1} \\
\hline I=e_{1} e_{2} e_{3} & I i=e_{1} & I j=e_{2} & I k=e_{3} \\
\hline
\end{array}
$$

\section{Background: Quaternions, Hyperquaternions and Multivectors}

In this section, we briefly introduce quaternions, hyperquaternions and multivectors $[7,11-15]$. The quaternion algebra $\mathbb{H}$ which contains $\mathbb{R}$ and $\mathbb{C}$ as particular cases is constituted by quaternions

$$
a=a_{1}+a_{2} i+a_{3} j+a_{4} k \quad\left(a_{i} \in \mathbb{R}\right)
$$

where $i, j, k$ multiply according to

$$
i^{2}=j^{2}=k^{2}=i j k=-1, i j=-j i=k, \text { etc. }
$$

The product of two quaternions $a, b$ is given by

$$
\begin{aligned}
a b= & \left(a_{1} b_{1}-a_{2} b_{2}-a_{3} b_{3}-a_{4} b_{4}\right)+\left(a_{1} b_{2}+a_{2} b_{1}+a_{3} b_{4}-a_{4} b_{3}\right) i \\
& +\left(a_{1} b_{3}+a_{3} b_{1}+a_{4} b_{2}-a_{2} b_{4}\right) j+\left(a_{1} b_{4}+a_{4} b_{1}+a_{2} b_{3}-a_{3} b_{2}\right) k .
\end{aligned}
$$

The conjugate of a quaternion is $a_{c}=a_{1}-a_{2} i-a_{3} j-a_{4} k$ with

$$
a a_{c}=a_{1}^{2}+a_{2}^{2}+a_{3}^{2}+a_{4}^{2},(a b)_{c}=b_{c} a_{c}
$$

The hyperquaternion algebra (over $\mathbb{R}$ ) is defined as the tensor product of quaternion algebras (or a subalgebra thereof). Examples of hyperquaternion algebras are the quaternions $\mathbb{H}$, tetraquaternions $\mathbb{H} \otimes \mathbb{H}$ and so on $\mathbb{H} \otimes \mathbb{H} \otimes \ldots \otimes \mathbb{H}$; subalgebras are the complex numbers $\mathbb{C}$, biquaternions $\mathbb{H} \otimes \mathbb{C}$, Dirac algebra $\mathbb{H} \otimes \mathbb{H} \otimes \mathbb{C}$, etc..

Calling $(i, j, k)$ the first quaternionic system, $(I, J, K)$ the second one and $(l, m, n)$ the third one, all systems commuting with each other, one has

$$
i \otimes i \otimes i=i I l, \quad i \otimes j \otimes k=i J n, \text { etc. }
$$

which uniquely defines the multiplication.

Hyperquaternions having $n$ generators $e_{i}$ such that $e_{i} e_{j}+e_{j} e_{i}=0(i \neq j)$, $e_{i}^{2}= \pm 1$ constitute Clifford algebras $C_{n}$. The choice of the generators entails a multivector structure as shown, in the case of biquaternions, in Table 1 . The $2^{n}$ elements of the algebra are composed of scalars, vectors $e_{i}$, bivectors $e_{i} e_{j}$, trivectors $e_{i} e_{j} e_{k}$ etc. yielding respectively the multivector spaces $V_{0}, V_{1}, V_{2}, V_{3}, \ldots V_{n}$. $C^{+}$is the subalgebra constituted by products of an even number of $e_{i}, C^{-}$is the rest of the algebra. The multivector structure allows to define basic operations like conjugation, duality and the interior and exterior products. 
Considering a general element $A$ of the algebra, the conjugate $A_{c}$ is obtained by replacing the $e_{i}$ by their opposite $-e_{i}$ and reversing the order of the elements

$$
\left(A_{c}\right)_{c}=A,(A B)_{c}=\left(B_{c}\right)\left(A_{c}\right) .
$$

The dual of $A$ is $A^{*}=i_{d} A$ where $i_{d}=e_{1} \wedge e_{2} \ldots \wedge e_{n}$ (to be defined below) and the commutator of two hyperquaternions is

$$
[A, B]=\frac{1}{2}(A B-B A) .
$$

The interior and exterior products of two vectors $a, b$ are obtained as follows. From the identity

$$
2 a b=\lambda \lambda^{-1}[(a b+b a)+(a b-b a)]
$$

where $\lambda= \pm 1$ is a given coefficient (allowing to eventually change the sign of the metric), one defines

$$
2 a . b=\lambda^{-1}(a b+b a), 2 a \wedge b=\lambda^{-1}(a b-b a)
$$

which are respectively a scalar and a bivector. A multivector $A_{p}=a_{1} \wedge a_{2} \wedge \ldots \wedge a_{p}$ $(2 \leq p<n)$ where $a_{p}$ are vectors, is then defined by recurrence

$$
\begin{aligned}
2 a \cdot A_{p} & =\lambda^{-p}\left[a A_{p}-(-1)^{p} A_{p} a\right] \in V_{p-1} \\
2 a \wedge A_{p} & =\lambda^{-p}\left[a A_{2}+(-1)^{p} A_{2} a\right] \in V_{p+1}
\end{aligned}
$$

By definition, we take

$$
A_{p} . a \equiv(-1)^{p-1} a \cdot A_{p}, A_{p} \wedge a \equiv(-1)^{p} a \wedge A_{p} .
$$

An important property of the exterior product is its associativity.

Interior and exterior products between multivectors are defined by

$$
\begin{aligned}
A_{p} \wedge B_{q} & =a_{1} \wedge\left(a_{2} \wedge \ldots \wedge a_{p} \wedge B_{q}\right) \\
A_{p} . B_{q} & =\left(a_{1} \wedge \ldots \wedge a_{p-1}\right) .\left(a_{p} . B_{q}\right), \quad(p \leq q)
\end{aligned}
$$

with $A_{p} \cdot B_{q}=(-1)^{p(q+1)} B_{q} \cdot A_{p}$ [16]. In particular, we have the following useful formulas where $B i$ are bivectors and $V_{p}[A]$ the multivector part $V_{p}$ of $A$

$$
\begin{aligned}
B_{1} B_{2} & =B_{1} \cdot B_{2}+B_{1} \wedge B_{2}+\left[B_{1}, B_{2}\right] \\
B_{1} \wedge B_{2} & =V_{4}\left[B_{1} B_{2}\right] \\
B_{1} \wedge B_{2} \wedge B_{3} & =V_{6}\left[B_{1}\left(B_{2} \wedge B_{3}\right)\right] \\
B_{1} \cdot\left(B_{2} \wedge B_{3}\right) & =V_{2}\left[B_{1}\left(B_{2} \wedge B_{3}\right)\right] \\
\left(B_{1} \wedge B_{2}\right) .\left(B_{3} \wedge B_{4} \wedge B_{5}\right) & =V_{2}\left[\left(B_{1} \wedge B_{2}\right)\left(B_{3} \wedge B_{4} \wedge B_{5}\right)\right] .
\end{aligned}
$$

Hyperquaternions yield all real, complex and quaternionic square matrices as well as the transposition, adjunction and transpose quaternion conjugate via a hyperconjugation defined as $\mathbb{H}_{c} \otimes \mathbb{H}_{c} \otimes \ldots \otimes \mathbb{H}_{c}$ as indicated in Table 2. 
Table 2. Hyperquaternions and matrices

\begin{tabular}{l|l}
$\mathbb{H} \otimes \mathbb{H} \simeq m(4, \mathbb{R})$ & $\mathbb{H}_{c} \otimes \mathbb{H}_{c} \simeq[m(4, \mathbb{R})]^{t}$ \\
\hline $\mathbb{H} \otimes \mathbb{H} \otimes \mathbb{C} \simeq m(4, \mathbb{C})$ & $\mathbb{H}_{c} \otimes \mathbb{H}_{c} \otimes \mathbb{C}_{c} \simeq[m(4, \mathbb{C})]^{\dagger}$ \\
$\mathbb{H} \otimes \mathbb{H} \otimes \mathbb{H} \simeq m(4, \mathbb{H})$ & $\mathbb{H}_{c} \otimes \mathbb{H}_{c} \otimes \mathbb{H}_{c} \simeq[m(4, \mathbb{H})]_{c}^{t}$
\end{tabular}

\section{Pseudo-Orthogonal Rotations}

In this section, we derive a hyperquaternion formulation of pseudo-euclidean rotations and develop a canonical decomposition. Historically, the formula of $n$ dimensional euclidean rotations $x^{\prime}=a x a^{-1}\left(a \in C_{n}^{+}\right)$was given by Lipschitz [17] and Moore developed a canonical decomposition thereof [8,9]. In this section we introduce, as an extension of Moore's method, within the hyperquaternion Clifford algebra framework, a canonical decomposition of pseudo-euclidean rotations and the Poincaré groups. After a brief review of the basic definitions and the Cartan theorem, we develop the canonical decomposition.

\subsection{Definitions and Theorem}

Let $C_{p, q}$ be a hyperquaternion algebra having $n=p+q$ generators $e_{i}$ and the quadratic form

$$
\begin{aligned}
x . y & =x_{1} y_{1}+\ldots+x_{p} y_{p}-\left(x_{p+1} y_{p+1} \ldots-x_{p+q} y_{p+q}\right) \\
& =\lambda^{-1}(x y+y x) / 2
\end{aligned}
$$

where $x, y$ are vectors $\left(x=x_{i} e_{i}\right)$. A vector $x$ is timelike if $x . x>0$, spacelike if $x . x<0$ and isotropic if $x . x=0$.

An orthogonal symmetry with respect to a plane going through the origin and perpendicular to a unit vector $a\left(a^{2}= \pm 1\right)$ is given by $[12,13]$

$$
x^{\prime}= \pm a x a
$$

with $x^{\prime} x^{\prime}=( \pm a x a)( \pm a x a)=x x$.

Definition 1. The pseudo-orthogonal group $O(p, q)$ is the group of linear operators which leave invariant the form $x \cdot y$.

Theorem 1. Every rotation of $O(p, q)$ is the product of an even number $2 m \leq n$ of symmetries.

Definition 2. The special orthogonal group $\mathrm{SO}^{+}(p, q)$ is constituted by rotations which preserve the orientation of the space of positive norm vectors and the space of negative norm vectors. 
A rotation of $S O^{+}(p, q)$ can thus be expressed as

$$
x^{\prime}=a x a_{c} \quad\left(a a_{c}=1\right)
$$

with $a=a_{1} a_{2} \ldots a_{2 m}, \in C^{+}$, where $a_{i}$ are unit vectors (with an even number of timelike and spacelike vectors). Developing the product (with $\lambda=1$ )

$$
a_{i} a_{j}=a_{i} . a_{j}+a_{i} \wedge a_{j}
$$

one sees that it contains a simple plane $B=a_{i} \wedge a_{j}$ such that $B^{2}=B . B+$ $B \wedge B$ is a scalar since $B \wedge B=0$. Hence, a rotation involves at most $m \leq n / 2$ simple planes. A canonical decomposition of rotations is obtained by choosing these simple planes to be orthogonal.

\subsection{Canonical Decomposition}

A rotation of $\mathrm{SO}^{+}(p, q)$ can be decomposed as

$$
a=e^{\frac{\Phi_{1}}{2} B_{1}} e^{\frac{\Phi_{2}}{2} B_{2}} \ldots e^{\frac{\Phi_{m}}{2} B_{m}} \quad\left(a a_{c}=1\right)
$$

where $B_{i}$ are $m$ simple orthogonal commuting planes such that $B_{i}^{2}= \pm 1$ together for $i \neq j$

$$
B_{i} \cdot B_{j}=0, B_{i} B_{j}=B_{j} B_{i}, B_{i} B_{j}=B_{i} \wedge B_{j} ;
$$

$\Phi_{i}$ are the angles of rotation within the planes $B_{i}$. According to whether $B_{i}^{2}=-1$ or $B_{i}^{2}=1$, one has respectively

$$
e^{\frac{\Phi_{i}}{2} B_{i}}=\cos \frac{\Phi_{i}}{2}+\sin \frac{\Phi_{i}}{2} B_{i}, e^{\frac{\Phi_{i}}{2} B_{i}}=\cosh \frac{\Phi_{i}}{2}+\sinh \frac{\Phi_{i}}{2} B_{i} .
$$

The rotation can be developed as

$$
a=S\left(1+b_{1} B_{1}\right)\left(1+b_{2} B_{2}\right) \ldots\left(1+b_{m} B_{m}\right)
$$

with $b_{i}=\tan \frac{\Phi_{i}}{2}$ (or $\tanh \frac{\Phi_{i}}{2}$ ). Since $a a_{c}=1$ one has

$$
\begin{gathered}
S^{2}\left(1+b_{1}^{2}\right)\left(1-b_{2}^{2}\right)\left(1-b_{3}^{2}\right)=1 \\
S=\frac{1}{\sqrt{\left(1 \pm b_{1}^{2}\right) \ldots\left(1 \pm b_{m}^{2}\right)}}
\end{gathered}
$$

which shows that $S$ is determined by the $b_{i}$. Writing

$$
B=b_{1} B_{1}+b_{2} B_{2}+b_{3} B_{3}
$$

one can express $a$ as

$$
a=S\left(1+B+\frac{B \wedge B}{2 ! S^{2}}+\ldots \frac{B \wedge B \wedge B \wedge \ldots(m \text { terms })}{m ! S^{m}}\right)
$$


which shows that the bivector $B$ determines completely the rotation.

If the scalar is nil, for example if $\left(\Phi_{1}= \pm \pi, B_{1}^{2}=-1\right)$, then $a$ is proportional to $B_{1}$

$$
a=B_{1} e^{\frac{\Phi_{2}}{2} B_{2}} e^{\frac{\Phi_{3}}{2} B_{3}} ;
$$

one then computes $B_{1}^{-1} a$ and comes back to the general expression to evaluate the remaining $b_{i}$ and $B_{i}$.

To determine the $b_{i}$ and $B_{i}$, one makes a change of variable $X_{i}=b_{i} B_{i}, x_{i}=$ $X_{i}^{2}= \pm b_{i}^{2}$ and considers the linear system of equations in $X_{i}[9]$

$$
\begin{aligned}
P_{1}= & B=\sum_{i=1}^{m} X_{i} \\
P_{2}= & (B \wedge B) . B=2 \sum_{i, j=1}^{m} X_{i} x_{j}(i \neq j) \\
P_{3}= & (B \wedge B \wedge B) .(B \wedge B)=3 ! 2 ! \sum_{i, j, k=1}^{m} X_{i} x_{j} x_{k} \quad(i \neq j, j<k) \\
& \ldots \ldots \\
P_{m}= & (B \wedge B \wedge \ldots m \text { factors }) .(B \wedge B \ldots(m-1) \text { factors }) \\
= & m !(m-1) ! \sum_{i=1}^{m} x_{1} x_{2} \ldots x_{i-1} x_{i+1} \ldots x_{m} X_{i} .
\end{aligned}
$$

The determinant $\Delta$ is the product

$$
\Delta=\left\{m ![(m-1) !]^{2}[(m-2) !]^{2} \ldots 1\right\} \prod_{i, j=1}^{m}\left(x_{i}-x_{j}\right) \quad(i \neq j, i<j) .
$$

If $\Delta \neq 0$, one obtains the bivectors $X_{i}$ as a function of $P_{m}$ and $x_{i}$. To determine the $x_{i}$, one writes the equations

$$
\begin{aligned}
S_{1}= & P_{1} \cdot P_{1}=\sum_{i=1}^{m} x_{i} \\
S_{2}= & P_{2} \cdot P_{1}=2 ! \sum_{i, j=1}^{m} x_{i} x_{j}(i \neq j) \\
S_{3}= & P_{3} \cdot P_{1}=(3 !)^{2} \sum_{i, j, k=1}^{m} x_{i} x_{j} x_{k} \quad(i \neq j, j<k) \\
& \ldots \ldots \\
S_{m}= & P_{m} \cdot P_{1}=(m !)^{2}\left(x_{1} x_{2} \ldots x_{m}\right) .
\end{aligned}
$$

The solutions yield $x_{i}= \pm b_{i}^{2}$, thus one obtains $b_{i}$ and $B_{i}$

$$
b_{i}=\sqrt{\left|x_{i}\right|}, B_{i}=\frac{X_{i}}{b_{i}} .
$$


If $\Delta=0$, the equations (36-41) are not independent, the $B$ bivector can nevertheless be decomposed in $m$ mutually orthogonal simple planes but this decomposition is not unique.

\section{Poincaré group in $n$ dimensions (via dual hyperquaternions)}

Much of physics being covariant with respect to the $4 D$ Poincaré group, we provide here a hyperquaternion representation of the $n D$ Poincaré groups in terms of dual hyperquaternions. Thereby one comes back to a $(n+1) D$ rotation which one can be decomposed canonically. The procedure is illustrated by a $5 D$ case (for example a color image with 2 spatial and 3 color dimensions) which might be of interest in machine learning [10]).

\subsection{General formalism}

The Poincaré group of the pseudo-euclidean space associated with the Clifford algebra $C_{p, q}(n=p+q)$ is constituted by the isometries of the metric

$$
d s^{2}=\left(d x_{1}^{2}+\ldots+d x_{p}^{2}\right)-\left(d x_{p+1}^{2}+\ldots+d x_{p+q}^{2}\right) .
$$

It includes the rotations $\mathrm{SO}^{+}(p, q)$, translations and reflections (time or spacelike). The reflections having already been dealt with above, we shall focus on the rotations and translations.

Consider a hyperquaternion algebra $\mathbb{H} \otimes \mathbb{H} \ldots \otimes \mathbb{H}$ (or a subalgebra thereof) with $n+1$ generators $e_{1}, e_{2}, \ldots e_{n}, e_{n+1}$ and let $X$ be a dual vector such that

$$
X=e_{n+1}+\varepsilon x
$$

where $x$ belongs to the vector space $V_{1}$ with $x=\sum_{i=1}^{n} e_{i} x_{i}\left(x_{i} \in \mathbb{R}\right)$ and $\varepsilon^{2}=0$ ( $\varepsilon$ commuting with $e_{i}$ ). An $n D$ hyperbolic rotation in $V_{1}$ leaves the last variable unchanged. Hence,

$$
X^{\prime}=a X a_{c}=e_{n+1}+\varepsilon x^{\prime}
$$

with $x^{\prime}=a x a_{c}, x^{\prime} x_{c}^{\prime}=x x_{c}, a a_{c}=1$. A translation in $V_{1}$ can be expressed as

$$
X^{\prime}=b X b_{c}
$$

with

$$
b=e^{\varepsilon e_{n+1} \frac{t}{2}}=1+\varepsilon e_{n+1} \frac{t}{2},\left(t=\sum_{i=1}^{n} e_{i} t_{i}, t_{i} \in \mathbb{R}\right)
$$

and $b b_{c}=1$. Developing Eq. (52), one obtains, assuming $e_{n+1}^{2}=-1$

$$
\begin{aligned}
X^{\prime} & =\left(1+\varepsilon e_{n+1} \frac{t}{2}\right)\left(e_{n+1}+\varepsilon x\right)\left(1-\varepsilon e_{n+1} \frac{t}{2}\right) \\
& =e_{n+1}+\varepsilon x-\varepsilon e_{n+1} e_{n+1} \frac{t}{2}-\varepsilon e_{n+1} e_{n+1} \frac{t}{2} \\
& =e_{n+1}+\varepsilon(x+t)
\end{aligned}
$$


which is a translation on the variables $1 \ldots n$ (if $e_{n+1}^{2}=1$, one simply takes $\left.b=e^{\varepsilon \frac{t}{2} e_{n+1}}\right)$. A combination of an $n D$ rotation and translation gives with $f=a b$ (or $b a$ )

$$
X^{\prime}=f X f_{c} \quad\left(f f_{c}=1, f \in C^{+}\right)
$$

which can be viewed as a a particular $(n+1) D$ rotation. One thus obtains a hyperquaternion representation of the Poincaré groups, distinct from the matrix one. A canonical decomposition leads to simple dual planes as will be illustrated in the following example.

\subsection{Example: 5D Poincaré group}

As application consider a $5 D$-space (for example a $2 D$ color image) imbedded in the $6 D$ hyperquaternion algebra $\mathbb{H} \otimes \mathbb{H} \otimes \mathbb{H}$ having six generators (see Appendix)

$$
e_{1}=k I, e_{2}=k J, e_{3}=k K l, e_{4}=k K m, e_{5}=k K n, e_{6}=j
$$

with the generic vector $X=e_{6}+\varepsilon x \quad\left(x=\sum_{i=1}^{5} e_{i} x_{i}\right)$. The transformation $X^{\prime}=f X f_{c}$ with

$$
\begin{aligned}
f & =e^{\frac{\Phi_{2}}{2} J l} e^{\varepsilon i(2 I+K n)} e^{\frac{\Phi_{1}}{2} I(m+n)} \\
& =(2+\sqrt{3} J l)[1+\varepsilon i(2 I+K n)]\left[\sqrt{3}+\sqrt{2} I\left(\frac{m}{\sqrt{2}}+\frac{n}{\sqrt{2}}\right)\right]
\end{aligned}
$$

and $\tanh \frac{\Phi_{1}}{2}=\sqrt{\frac{2}{3}}\left(=b_{1}\right), \tanh \frac{\Phi_{2}}{2}=\frac{\sqrt{3}}{2}\left(=b_{2}\right)$ is a $5 D$-Poincaré transform. Applying the canonical decomposition presented above, one obtains

$$
f=e^{\frac{\Phi_{2}}{2} B_{2}} e^{X_{3}} e^{\frac{\Phi_{1}}{2} B_{1}}
$$

with the same values of $\Phi_{1}, \Phi_{2}$ as above and the following simple commuting orthogonal dual planes $B_{1}, B_{2}, X_{3}$

$$
\begin{aligned}
& B_{1}=\frac{1}{\sqrt{2}} I(m+n)+\varepsilon \frac{1}{\sqrt{2}}\left[\frac{\sqrt{3}}{2} K(m+n)-i J\right] \\
& B_{2}=J l+2 \varepsilon i\left(\frac{2}{\sqrt{3}} I-K l\right) \\
& X_{3}=\frac{\varepsilon}{2} i K(-m+n) .
\end{aligned}
$$

with $\left(B_{1}\right)^{2}=\left(B_{2}\right)^{2}=1,\left(X_{3}\right)^{2}=0$.

\section{Conclusion}

The paper has given a hyperquaternion representation of pseudo-euclidean rotations and the Poincaré groups in $n$ dimensions, distinct from the matrix one. A canonical decomposition of these groups was introduced, as an extension of an euclidean formalism, within a hyperquaternion Clifford algebra framework and illustrated by a $5 D$ example. Potential geometric applications include in particular, moving reference frames and machine learning. 


\section{Acknowledgements}

This work was supported by the LABEX PRIMES (ANR-11-LABX-0063) and was performed within the framework of the LABEX CELYA (ANR-10-LABX0060) of Université de Lyon, within the program "Investissements d'Avenir" (ANR11-IDEX-0007) operated by the French National Research Agency (ANR).

\section{A Multivector structure of $\mathbb{H} \otimes \mathbb{H} \otimes \mathbb{H}$}

$$
\begin{aligned}
& {\left[\begin{array}{llll}
1 & l=e_{4} e_{5} & m=e_{5} e_{3} & n=e_{3} e_{4} \\
I=e_{2} e_{3} e_{4} e_{5} & I l=e_{3} e_{2} & I m=e_{4} e_{2} & I n=e_{5} e_{2} \\
J=e_{3} e_{1} e_{4} e_{5} & J l=e_{1} e_{3} & J m=e_{1} e_{4} & J n=e_{1} e_{5} \\
K=e_{2} e_{1} & K l=e_{2} e_{1} e_{4} e_{5} & K m=e_{1} e_{2} e_{3} e_{5} & K n=e_{2} e_{1} e_{3} e_{4}
\end{array}\right]} \\
& +i\left[\begin{array}{llll}
1=e_{1} e_{2} e_{3} e_{4} e_{5} e_{6} & l=e_{2} e_{1} e_{3} e_{6} & m=e_{2} e_{1} e_{4} e_{6} & n=e_{2} e_{1} e_{5} e_{6} \\
I=e_{6} e_{1} & I l=e_{4} e_{1} e_{5} e_{6} & I m=e_{5} e_{1} e_{3} e_{6} & I n=e_{3} e_{1} e_{4} e_{6} \\
J=e_{6} e_{2} & J l=e_{4} e_{2} e_{5} e_{6} & J m=e_{5} e_{2} e_{3} e_{6} & J n=e_{3} e_{2} e_{4} e_{6} \\
K=e_{3} e_{4} e_{5} e_{6} & K l=e_{6} e_{3} & K m=e_{6} e_{4} & K n=e_{6} e_{5}
\end{array}\right] \\
& +j\left[\begin{array}{llll}
1=e_{6} & l=e_{4} e_{5} e_{6} & m=e_{6} e_{5} e_{3} & n=e_{3} e_{4} e_{6} \\
I=e_{2} e_{3} e_{4} e_{5} e_{6} & I l=e_{3} e_{2} e_{6} & I m=e_{6} e_{4} e_{2} & I n=e_{6} e_{5} e_{2} \\
J=e_{4} e_{3} e_{5} e_{6} e_{1} & J l=e_{1} e_{3} e_{6} & J m=e_{1} e_{4} e_{6} & J n=e_{1} e_{5} e_{6} \\
K=e_{2} e_{1} e_{6} & K l=e_{2} e_{1} e_{4} e_{5} e_{6} & K m=e_{1} e_{2} e_{3} e_{5} e_{6} & K n=e_{2} e_{1} e_{3} e_{4} e_{6}
\end{array}\right] \\
& +k\left[\begin{array}{llll}
1=e_{2} e_{1} e_{3} e_{4} e_{5} & l=e_{1} e_{2} e_{3} & m=e_{1} e_{2} e_{4} & n=e_{1} e_{2} e_{5} \\
I=e_{1} & I l=e_{1} e_{4} e_{5} & I m=e_{3} e_{1} e_{5} & I n=e_{1} e_{3} e_{4} \\
J=e_{2} & J l=e_{2} e_{4} e_{5} & J m=e_{3} e_{2} e_{5} & J n=e_{2} e_{3} e_{4} \\
K=e_{4} e_{3} e_{5} & K l=e_{3} & K m=e_{4} & K n=e_{5}
\end{array}\right]
\end{aligned}
$$

\section{References}

1. Ungar, A.: Beyond Pseudo-Rotations in Pseudo-Euclidean Spaces. Academic Press, London (2018)

2. Ferreira, M., Sommen, F.: Complex Boosts: A Hermitian Clifford Algebra Approach. Advances in Applied Clifford Algebras, 23: 339-362 (2013)

3. Lounesto, P.: Clifford Algebras and Spinors. Cambridge University Press, Cambridge (2001)

4. Crumeyrolle, A.: Orthogonal and Symplectic Clifford Algebras: Spinor Structures. Kluwer Academic Publishers, Dordrecht (1990)

5. Perez-Gracia, A., Thomas, F. On Cayley's Factorization of $4 D$ Rotations and Applications. Advances in Applied Clifford Algebras, 27:523-538 (2017)

6. Richard, A., Fuchs, L., Andres, E., Largeteau-Skapin, G. Decomposition of nD-rotations: Classification, properties and algorithm. Graphical Models, Elsevier, 73 (6), pp. 346-353 (2011).

7. Girard, P.R., Clarysse, P., Pujol, R., A.,Goutte, R, Delachartre, P.: Hyperquaternions: A New Tool for Physics. Advances in Applied Clifford Algebras, 28:68 (2018) 
8. Moore, C.L.E.: Hyperquaternions. Journal of Mathematics and Physics, vol. 1, pp. 63-77 (1922)

9. Moore, C.L.E.: Rotations in Hyperspace. Proceedings of the American Academy of Arts and Sciences, vol. 53, No. 8, pp. 651-694 (1918)

10. Xuanyu Zhu, Yi Xu, Hongteng Xu, Changuian Chen Quaternion Convolutional Neural Networks. ECCV, 2018.

11. Girard, P.R., Clarysse, P., Pujol, R., A., Wang, L, Delachartre, P.: Differential Geometry Revisited by Biquaternion Clifford Algebra. In J.-D. Boissonnat et al (Eds.): Curves and Surfaces. Springer (2015)

12. Girard, P.R.: Quaternions, Clifford Algebras and Relativistic Physics. Birkhäuser, Basel (2007)

13. Girard, P.R.: Algèbre de Clifford et Physique relativiste . PPUR, Lausanne (2004)

14. Girard, P.R.: Einstein's equations and Clifford algebra. Advances in Applied Clifford Algebras, vol. 9, 2, pp. 225-230 (1999)

15. GiRARD, P.R.: The quaternion group and modern physics. Eur. J. Phys., vol. 5, pp. 25-32 (1984)

16. Casanova, G.: L'algèbre vectorielle. PUF, Paris (1976)

17. Lipschitz, R.: Principes d'un calcul algébrique qui contient comme espèces particulières le calcul des quantités imaginaires et des quaternions. C.R. Acad. Sci. Paris, vol. 91, pp. 619-621, 660-664 (1880) 